\title{
Can anticoagulation care be improved with technology?
}

\author{
Jerry H. Gurwitz MD
}

$\infty \infty$

See related research paper by Oake and colleagues, page 927

I $\mathrm{n}$ this issue of CMAJ, Oake and colleagues ${ }^{1}$ report the results of an observational study of an interactive voice response system to assist in managing the care of low-risk patients receiving warfarin. The system communicated results of international normalized ratio testing, dosage schedules and dates of subsequent tests to patients. It also reminded them of upcoming and missed appointments. The authors reported that, although the quality of oral anticoagulant therapy did not change, the intervention modestly improved the efficiency of providing care. The efforts of Oake and colleagues lay the groundwork for more sophisticated technology-based approaches to improve the care of a broader range of patients receiving anticoagulant therapy.

That the quality of anticoagulation control was not improved by this intervention is no surprise. The authors emphasized that they were purposeful in wanting "to evaluate the effectiveness of the interactive voice response system under optimal conditions." Patients who had unstable anticoagulation control were excluded. Before enrolment, the study participants had been in the therapeutic range nearly $80 \%$ of the time. This compares with a therapeutic range achieved less than $70 \%$ of the time, even for patients managed in the most ideal of settings, such as a well-run clinical trial or a highly experienced anticoagulation clinic. ${ }^{2}$ In addition, in the context of the study by Oakes and colleagues, no hemorrhagic or thromboembolic events occurred over 135 patient-years of exposure to oral anticoagulant therapy. In summary, this was a nearly perfectly managed group of patients, both before and throughout the intervention period. Such patients comprise a special subset who would probably do well on warfarin therapy under almost any circumstances.

Efforts to improve the quality of care ideally focus on situations in which there are special challenges in caring for patients, the adverse consequences of suboptimal care are well established, and the benefits of improving care are unequivocal. Such efforts often focus on patients at highest risk. In this respect, the work of Oake and colleagues presents a paradox of sorts: the study participants were extraordinarily well managed, even before the intervention, and they were at very low risk of bleeding complications. Is there any justification for targeting these patients with a technology-based intervention?

Although not fully considered by the authors, their findings do suggest a number of possibilities for improving the effectiveness and efficiency of managing large numbers of patients receiving oral anticoagulant therapy. For example, use of the interactive voice response system may be an ideal strategy to increase staff time to address the special needs of more challenging patients. The modest reduction in workload

\section{Key points}

- Technology-based efforts to improve quality are often focused on high-risk patients, yet the study by Oake and colleagues focused on low-risk patients receiving warfarin therapy.

- Although the quality of anticoagulation therapy did not change with use of the interactive voice response system, some modest efficiencies in providing care were achieved.

- There are important opportunities to refine and expand this technology-based intervention, and to determine its effectiveness in high-risk patients.

documented in this study might be increased many times over, as the number of stable patients managed by an anticoagulation service increases. Furthermore, additional efficiencies would be gained by directly connecting the laboratory to the computerized decision support system, eliminating clerical duties performed by professional staff. In the scenario described by Oake and colleagues, the laboratory forwarded the results of testing to the clinic, and a pharmacist manually entered the results into each patient's profile in the computerized decision support system and then approved or changed the recommended dose and the next test date. With additional improvements in algorithms for determining the appropriate dose of warfarin, ${ }^{3}$ the checking function of the pharmacist might be eliminated in many instances, and there could be a seamless connection between the decision support system and the interactive voice response system. This refinement would provide additional time for the pharmacist to focus on the most complex patients.

The work by Oake and colleagues lays the groundwork for even more sophisticated technology-based approaches to oral anticoagulant management. As Green and colleagues have written, ${ }^{4}$ "electronic medical records and secure patient websites increasingly let patients view portions of their medical record, access health care services, and communicate with their health care team online." Web-based care may be suitable for managing the care of patients taking warfarin therapy, particularly those who self-monitor. Web-based care could also overcome the problems of telephone communication faced by patients with impaired hearing and address concerns of leaving messages about test results and dosage instructions on telephone answering machines.

In summary, the study by Oake and colleagues represents an important contribution to ongoing efforts to improve the

Jerry Gurwitz is Executive Director of the Meyers Primary Care Institute and Chief of the Division of Geriatric Medicine at the University of Massachusetts Medical School, Worcester, USA. 
quality and efficiency of anticoagulation care. There are important opportunities to refine and expand this technologybased intervention. Future research should use randomized controlled trial designs. In addition, efforts should be made to enrol "less-than-ideal" participants, who may be more generalizable to the broader patient population receiving warfarin therapy.

Perhaps the greatest strength of any research study lies in the questions that it generates and the foundation that it lays for future investigative efforts. In this regard, there is nothing at all paradoxical about the work of Oake and colleagues.

Competing interests: None declared.

\section{REFERENCES}

1. Oake N, van Walraven C, Rodger MA, et al. Effect of an interactive voice response system on oral anticoagulant management. CMAJ 2009;180:927-33.

2. van Walraven C, Oake N, Wells PS, et al. Burden of potentially avoidable anticoagulant-associated hemorrhagic and thromobembolic events in the elderly. Chest 2007;131:1508-15.

3. International Warfarin Pharmacogenetics Consortium. Estimation of the warfarin dose with clinical and pharmacogenetic data. N Engl J Med 2009;360:753-64.

4. Green BB, Cook AJ, Ralston JD, et al. Effectiveness of home blood pressure monitoring, web communication, and pharmacist care on hypertension control: a randomized controlled trial. JAMA 2008;299:2857-67.

Correspondence to: Dr. Jerry H. Gurwitz, Meyers Primary Care Institute, 630 Plantation St., Worcester MA 01605, USA; fax 508 595-2200; jerry.gurwitz@umassmed.edu

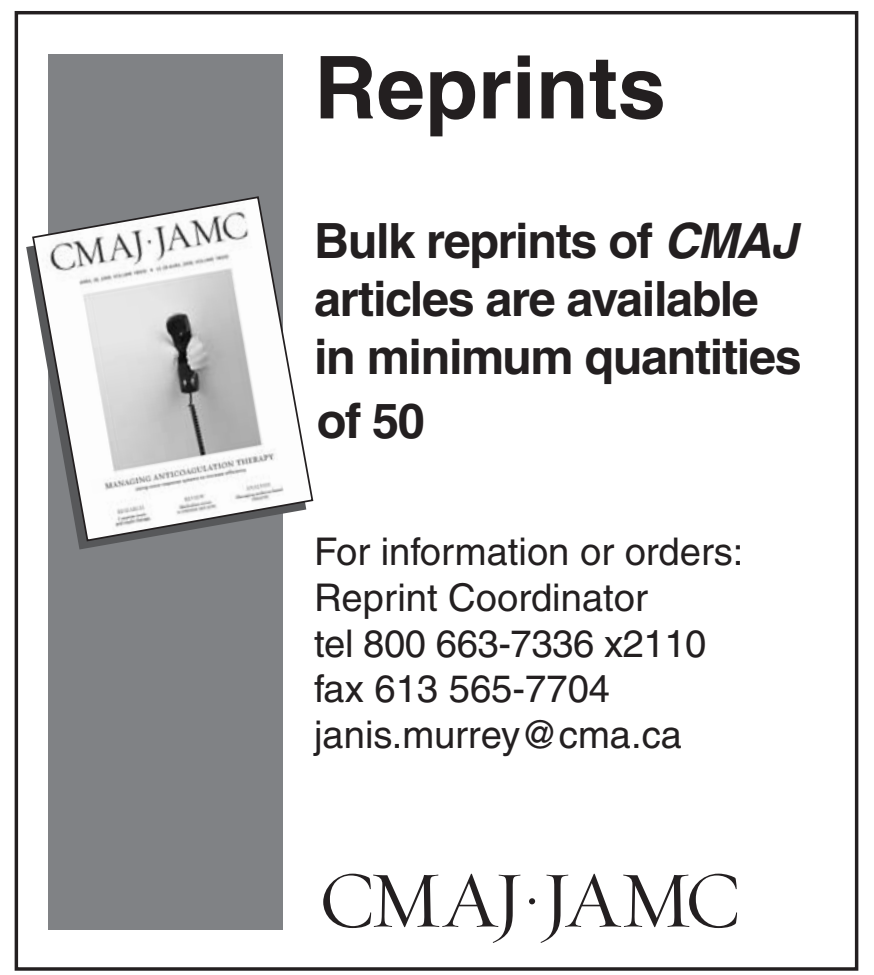

